\title{
Uso de suelo y biomateriales
}

Recibido: 26 Novembro 2007 / Aceptado: 27 Outubro 2008 (C) IBADER- Universidade de Santiago de Compostela 2008

Resumen A la hora de diseñar un producto realizado con materiales biodegradables, se deben tener en cuenta todos los impactos asociados a lo largo de su ciclo de vida. La mayoría de los Análisis de Ciclo de Vida (ACV) existentes sobre biopolímeros no se han ocupado de la categoría de impacto de uso del suelo. Dado que la fracción orgánica de los productos biodegradables proviene normalmente de residuos agrícolas, esta categoría surge como una cuestión medioambiental primordial debido a los impactos que producen los cultivos.

En el caso de que los biopolímeros tuvieran un gran éxito en el mercado, probablemente surgiría la necesidad de plantar nuevos campos de cultivo para cubrir la demanda, causando así nuevos impactos medioambientales derivados del uso del suelo.

En este estudio se hace una comparación de las metodologías disponibles para evaluar dichos impactos, empleando distintas fuentes de datos para su aplicación. Para ello se evalúa el impacto causado por una hectárea de cultivo de patatas en dos zonas de España con diferentes características: los bosques mediterráneos del Sistema Ibérico y los pastos de la región cantábrica.

Los resultados vienen a mostrar que no se han desarrollado aún metodologías fiables para evaluar esta categoría de impacto.

Palabras clave ACV · uso de suelo · biomateriales · cultivos

Abstract When designing a product made of biodegradable materials, all of the impacts associated with the product along its life cycle should be taken into account. Existing Life Cycle Assessments (LCA) studies of biopolymers have often

Daniel Garraín · Rosario Vidal · Vicente Franco Grupo de Ingeniería del Diseño (GID), Dpto. Ingeniería Mecánica y Construcción, Universitat Jaume I.

Avda. Sos Baynat s/n, E-12071 Castellón (España). Tel. +34964729252, Fax +34964728106

e-mail: garrain@uji.es neglected the land use impact category. Given that the organic fraction of biodegradable products often comes from agricultural waste, this category arises as an essential environmental issue due to the impacts of crops.

Should biodegradable materials have great market success, it is possible that additional land would have to be transformed into crop fields, therefore causing environmental impacts from land use.

A comparison of the several methodologies available to assess this impact is carried out in this study, using several data source. In order to illustrate the disparity among the existing methods, the impact caused by the substitution of a certain type of land for a one-hectare potato crop field was assessed. Two areas in Spain possessing different soil characteristics were chosen. These were the Mediterranean forests of the Iberian mountain group and the pastures of the north-western Cantabrian region.

Results come to show that no reliable methodologies have yet been developed to assess land use.

Keywords LCA · land use · biomaterials · crop fields

\section{Introducción}

Según los estudios desarrollados por diversos autores (Gärtner y Reinhart, 2004; Braschkat y Gärtner, 2004; Patel et al., 2003; Scott, 2000; Känzig et al., 2003), las categorías de impacto más utilizadas en los ACV para evaluar el impacto medioambiental de plásticos y productos biodegradables son: calentamiento global, acidificación, eutrofización y agotamiento de recursos fósiles.

Cuando se diseña un producto realizado con materiales biodegradables, todos los impactos asociados a lo largo de su ciclo de vida deben ser tenidos en cuenta. El reemplazo de materiales convencionales por biomateriales conlleva impactos medioambientales que no son siempre evidentes ni fáciles de cuantificar. Los ACV existentes sobre biopolímeros no se han ocupado de la categoría de impacto de uso del suelo, con excepciones como Müller-Sämman et 
al. (2003), quienes reivindican la necesidad de desarrollar indicadores para la demanda de uso de suelo.

Dado que la fracción orgánica de los productos biodegradables proviene normalmente de residuos agrícolas, la categoría de uso de suelo surge como una cuestión medioambiental primordial debido a los impactos que producen los cultivos.

En los estudios destinados a evaluar el impacto ambiental de los productos biodegradables mediante la aplicación del $A C V$, la categoría de impacto sobre el uso del suelo no siempre se considera o no se adecua a la realización de dichas evaluaciones. Esta circunstancia, junto con la escasa disponibilidad de datos, ha motivado que la aplicación de los indicadores de uso del suelo resulte, todavía hoy en día, compleja debido a que existe una gran indefinición sobre los parámetros a considerar y la metodología a seguir.

Varios autores han revisado los diferentes indicadores para el cálculo del impacto del uso del suelo. En los primeros estudios, la evaluación se realizaba en base a la superficie de terreno ocupada. La categoría de impacto de uso del suelo se relaciona con el área ocupada, generalmente en combinación con el tiempo durante el cual se realizan las actividades humanas (Lindeijer, 2000a). Pero este valor de ocupación debe estar acompañado de una evaluación cualitativa de los cambios que sufre el suelo como consecuencia de su uso. Con carácter internacional se acepta que la fase de inventario de ciclo de vida debe centrarse en dos aspectos del uso del suelo: el área ocupada y los cambios que se producen en la calidad del suelo. Aparecen así dos conceptos: ocupación y transformación del suelo (Weidema y Lindeijer, 2001; Antón, 2007).

Estudios posteriores han desarrollado métodos para presentar indicadores que miden el deterioro de la biodiversidad, definida como la variedad de plantas y de vida animal en un hábitat particular, incluyendo la diversidad de ecosistemas (variedad de hábitats en una región o el mosaico de pequeñas parcelas encontrados dentro de un paisaje), diversidad genética (combinación de diferentes genes encontrados dentro de una población de especies singulares, y la pauta de variación encontrada dentro de diferentes poblaciones de una misma especie) y la diversidad de especies (variedad y abundancia de diferentes tipos de organismos que habitan un área determinada).

También se han desarrollado otros métodos que miden las alteraciones de las funciones de soporte vital del suelo debidas al uso del mismo. El suelo ejerce una serie de funciones que ayudan a mantener la vida en los ecosistemas, llamadas funciones de sostén o soporte vital. En los ecosistemas terrestres, la disponibilidad de recursos bióticos está relacionada con su potencial de productividad, entendida como la producción de biomasa fotosintetizada por las plantas verdes a partir de agua, CO2 y energía solar. Si a esta biomasa se le resta la consumida en la respiración celular, se obtiene la Producción Primaria Neta (Net Primary Production, NPP) (Antón, 2004). El valor de NPP puede considerarse como la expresión del valor natural y dinámico de un área determinada. Este valor es el escogido en los métodos de Weidema y Lindeijer (2001) y Lindeijer (2000b) como medida de la contribución al soporte vital, expresado en unidades de masa de carbono elemental por unidad de área y tiempo.

De entre estos métodos, cabe destacar los desarrollados por Köllner (2000, 2001), Goedkoop y Spriensma (2001), Vogtländer et al. (2004) y Müller-Wenk (1998) para el análisis del impacto del uso del suelo sobre la biodiversidad; los de Milà i Canals (2003) y Cowell y Clift (2000) para analizar los impactos sobre las funciones de soporte de vida; y los de Mattsson et al. (2000), Cowell (1998), Lindeijer (2000b) y Weidema y Lindeijer (2001), que evalúan los impactos sobre la biodiversidad y sobre las funciones de soporte vital por separado.

Por otra parte, algunos autores como Muys y García Quijano (2000) y Wagendorp et al. (2006) han desarrollado nuevos métodos para analizar el impacto debido al uso del suelo basados en la medida de la capacidad que posee un ecosistema para disipar la exergía solar.

Otros autores han realizado comparaciones cualitativas de los métodos que tienen en cuenta los impactos sobre la biodiversidad y las funciones de soporte de vida, como van der Voet (2001), o han aplicado algunos de ellos a casos particulares: materiales de construcción (Lindeijer et al., 2002), invernaderos (Antón et al., 2007) y cultivos para aceites vegetales (Mattson et al., 2000).

En el caso de que los materiales biopolímeros tuvieran un gran éxito en el mercado, probablemente surgiría la necesidad de plantar nuevos campos de cultivo para cubrir la demanda, causando así nuevos impactos medioambientales por el uso del suelo. Para ilustrar la disparidad existente entre los métodos actuales de evaluación de este tipo de impactos, se evalúa el impacto causado por una hectárea de cultivo de patatas en dos zonas de España con diferentes características y tipo de suelo: los bosques mediterráneos del Sistema Ibérico y los pastos de la región cantábrica.

\section{Material y métodos}

Los métodos seleccionados para esta comparativa han sido los destinados a evaluar el impacto sobre la biodiversidad y sobre las funciones de soporte de vida.

Para evaluar el impacto del uso del suelo sobre la biodiversidad se han aplicado los métodos de Köllner (2000), Goedkoop y Spriensma (2001), Vogtländer et al. (2004) y Weidema y Lindeijer (2001), debido a la amplia disponibilidad de datos para su aplicación. Estos métodos miden el número de especies de plantas vasculares, las cuales son representativas de la diversidad de especies en general. Los trabajos de Barthlott et al. $(1996,1999)$ han mostrado una fuerte correlación entre el número de especies de plantas vasculares y la abundancia de otras especies de animales y plantas. La razón principal de elegir la diversidad de plantas vasculares como el indicador para la biodiversidad radica en que se trata del único indicador para el que se poseen datos científicos globales disponibles a diferentes escalas (Lindeijer, 2000b). 
Para su aplicación se tienen que considerar correspondencias entre los tipos de suelo estudiados y los tipos clasificados en la lista de CORINE Land Cover 2000 (Disponible

en

http://terrestrial.eionet.europa.eu/CLC2000/classess [Febrero 2007]), tomando como la zona cantábrica equivalente a pastos menos intensivos (código 2.3.1.2), la zona del sistema ibérico equivalente a bosques (código 3.1.1) y el cultivo de patatas como cultivo convencional (código 2.2.1.1).

En caso particular, en el método de Weidema y Lindeijer (2001) se tuvieron en cuenta las siguientes características geográficas en cuanto a tipo de suelo, altitud sobre el nivel del mar y latitud aproximada: para la zona cantábrica el suelo se corresponde con "grassland", de 0 a 1000 metros de altitud y $40^{\circ}$ aproximados de latitud; para la zona del sistema ibérico el suelo se corresponde con "mixed forest", de 1000 a 3000 metros de altitud y también $40^{\circ}$ aproximados de latitud. El cultivo de patatas se corresponde con "convencional and permanent crop". Este método dista un poco de los otros considerados ya que mide la riqueza de especies vasculares ( $\mathrm{nSR}$ ) del ecosistema modificada por la escasez normalizada del ecosistema (nES), expresada como la inversa del área potencial que podría ser ocupada por el ecosistema si no existieran actividades humanas; y la vulnerabilidad normalizada del ecosistema $(\mathrm{nEV})$, que indica en número relativo de especies afectadas por un cambio en el área del ecosistema.

Para evaluar el impacto sobre las funciones de soporte de vida del suelo o sobre la productividad del mismo, se han aplicado los métodos de Weidema y Lindeijer (2001) y Lindeijer (2000b), utilizando distintas fuentes de datos.

\section{Resultados y discusión}

\section{Impacto del uso del suelo sobre la biodiversidad}

La tabla 1 muestra los resultados de la aplicación de las diferentes metodologías para el cálculo del impacto del uso del suelo sobre la biodiversidad. La última fila expresa la ratio entre los valores del indicador para cada zona.

A la luz de estos resultados, se puede considerar que, para un cambio en el uso del suelo, el impacto sobre la biodiversidad es mayor en los bosques del Sistema Ibérico, ya que en ellos habita un mayor número de especies de plantas vasculares. En los tres primeros métodos, las ratios entre los impactos que causa el reemplazo por un campo de cultivo en un área y otra son similares, resultando éste mayor en el Sistema Ibérico por factor aproximado de 8 . Esto es debido a que los tres métodos utilizan bases metodológicas comunes (Köllner, 2000). Sin embargo, la aplicación del método de Weidema y Lindeijer (2001) resulta en una ratio considerablemente diferente, próxima a 3. Esta diferencia podría atribuirse al uso de fuentes de datos diferentes y al hecho de que el método utiliza otras variables para evaluar el impacto, tales como la escasez y la vulnerabilidad de los ecosistemas citadas anteriormente.

Impacto del uso del suelo sobre las funciones de soporte vital

La primera fila de la tabla 2 muestra los resultados de la aplicación de los dos métodos seleccionados para el cálculo del impacto del uso del suelo sobre las funciones de soporte a la vida. Estos resultados, en términos de productividad, reflejan que los terrenos ocupados por cultivos de patatas son más productivos que los bosques en los casos donde la intervención humana modifica el valor de NPP, tal y como predecían los autores (Weidema y Lindeijer, 2001). Este incremento de la productividad es debido al uso de fertilizantes, abonos, irrigación, etc. Por el contrario, cuando un pasto es reemplazado por un campo de patatas, existe un impacto negativo sobre la productividad. Estos resultados opuestos a los obtenidos para el impacto del uso del suelo sobre la biodiversidad.

Los resultados de la aplicación de los métodos anteriores pueden variar significativamente en función de la fuente de datos utilizada para la obtención de los valores de NPP. Considerando los valores propuestos por Vitousek et al. (1986) y Margalef (1998), los resultados son muy dispares, como muestran las siguientes filas de la tabla 2.

\section{Conclusiones}

Vistos los resultados obtenidos, es necesaria la convergencia en el desarrollo de metodologías para evaluar el impacto del uso del suelo. En nuestro caso de estudio se muestra que no se han desarrollado todavía metodologías globales para analizar la categoría de impacto de ocupación

\begin{tabular}{ccccc}
\hline Método & Köllner (2000) & $\begin{array}{c}\text { Goedkoop y } \\
\text { Spriensma }(2001)\end{array}$ & $\begin{array}{c}\text { Vogtländer et al. } \\
(2004)\end{array}$ & $\begin{array}{c}\text { Weidema y } \\
\text { Lindeijer (2001) }\end{array}$ \\
\hline Indicador & $\begin{array}{c}\text { Daño potencial de } \\
\text { transformación } \\
\left(\Delta \mathrm{D}_{\mathrm{tr}}\right)\end{array}$ & $\begin{array}{c}\text { Calidad del } \\
\text { ecosistema de } \\
\text { conversión }\left(E Q_{\text {conv }}\right)\end{array}$ & $\begin{array}{c}\text { Indicador de } \\
\text { riqueza de } \\
\text { especies }(\Delta S R I)\end{array}$ & $\begin{array}{c}\text { Impacto de } \\
\text { ocupación (Iocc,bio) }\end{array}$ \\
\hline Unidades & $\mathrm{m}^{2} \cdot$ año & $\mathrm{m}^{2} \cdot$ año & $\mathrm{m}^{2}$ equivalente & Persona-eq. \\
\hline S. Ibérico & 78333 & 309000 & 1566 & 68 \\
\hline Z. Cantábrica & 10000 & 30000 & 200 & 24 \\
\hline Ratio & 7,8 & 10,3 & 7,8 & 2,8 \\
\hline
\end{tabular}

Tabla 1.- Resumen de resultados del impacto del uso del suelo sobre la biodiversidad por metodología para cada zona 


\begin{tabular}{ccccc}
\hline \multirow{2}{*}{$\begin{array}{c}\text { Fuente de datos NPP potencial } \\
\text { y real }\end{array}$} & \multicolumn{5}{c}{$\begin{array}{c}\text { Impacto de ocupación } \\
\text { (locc, proa) (persona-eq.) }\end{array}$} & $\begin{array}{c}\text { Cambio en el ecosistema } \\
(\mathrm{EC} L \mathrm{LS} \text { ) (kg Carbono/año) }\end{array}$ \\
\cline { 2 - 5 } & Z. Cantábrica & S. Ibérico & Z. Cantábrica & S. Ibérico \\
\hline Weidema y Lindeijer (2001) & 23 & -17 & 1150 & -650 \\
\hline Vitousek et al. (1986) & 38 & 66 & 1881 & 2538 \\
\hline $\begin{array}{c}\text { Vitousek et al. (1986) [NPP pot] y } \\
\text { Margalef (1998) [NPP real] }\end{array}$ & -33 & -26 & -1649 & -992 \\
\hline
\end{tabular}

Tabla 2.- Impacto del uso del suelo sobre la productividad para cada zona, por fuente de datos de obtención de NPP

y transformación del suelo, aun cuando se acepta que ésta es una categoría de impacto crucial dadas sus consecuencias sobre la calidad del suelo a largo plazo.

En Milà i Canals (2006) se constató la ausencia de un consenso claro acerca de la preferencia entre indicadores del número de especies vasculares o los de nivel de ecosistema. En este estudio se ha mostrado que la aplicación de ambos métodos para la evaluación de situaciones análogas puede llevar a resultados opuestos.

La aplicación del método de Weidema y Lindeijer (2001) produce resultados confusos a la hora de determinar si un cambio en el uso del suelo provoca un impacto medioambiental positivo o negativo. Comparando un cambio en el uso del suelo en dos zonas diferentes, puede producirse un mayor impacto sobre las funciones de soporte vital en una zona, mientras que la biodiversidad puede verse dañada de forma más significativa en la otra zona. Por esta razón, es necesaria una mejora en el desarrollo de los indicadores que tienen en cuenta ambos impactos sobre la biodiversidad y la productividad (o funciones de soporte vital) mediante la aplicación métodos multicriterio que combinen las bondades de ambos tipos de análisis. Además, la inconsistencia que existe entre las fuentes de datos disponibles -como se ha ilustrado en el ejemplo de las diversas fuentes para la obtención del valor de NPP. indica que es necesario un gran esfuerzo para conseguir unos datos más fiables y consistentes sobre las características de los suelos que faciliten el desarrollo de los indicadores necesarios.

Un aspecto importante del impacto del uso del suelo es la dependencia del lugar donde el cambio de uso tiene lugar. Por ello, sería necesario estandarizar los métodos para el análisis del impacto asociado al uso del suelo. Esta tarea podría comenzar con la identificación de indicadores de uso de suelo a nivel local y regionales, cuyos resultados podrían extrapolarse posteriormente a niveles superiores.

Como comentario final, se recomienda tomar en consideración las categorías de impacto que, como el uso del suelo, son consideradas con menor frecuencia en los análisis de ciclo de vida: el impacto estético, ruido, olor, etc., puesto que éstas contribuyen de manera significativa al impacto medioambiental de cualquier tipo de producto o proceso.

Agradecimientos Este trabajo se ha desarrollado como parte de los siguientes proyectos: "Análisis del ciclo de vida de residuos de materiales biodegradables y biocompuestos, como alternativa a los polímeros convencionales" (Programa Nacional de Ciencias
Medioambientales y Tecnología del Programa Nacional de I+D 2004-2007 del Ministerio de Medio Ambiente de España. Ref.: 566/2006/1-2.4) y "Desarrollo de categorías de impacto aplicadas a materiales cerámicos usando la metodologías del análisis del ciclo de vida" (Programa Nacional de Transporte y Construcción del Programa Nacional de Investigación Científica, Desarrollo e Innovación Tecnológica 2004-2007 del Ministerio de Fomento de España. Ref.: C46/2006).

\section{Bibliografía}

Antón, A. (2004) "Utilización del análisis del ciclo de vida en la evaluación del impacto ambiental del cultivo bajo invernadero mediterráneo". Tesis doctoral, Universitat Politécnica de Catalunya, Barcelona.

Antón, A., Castells, F., Montero, J.I. (2007) "Land use indicators in life cycle assessment. Case study: The environmental impact of Mediterranean greenhouses". Journal of Cleaner Production, 15 (2007), pp. 432-438.

Barthlott, W., Lauer, W., Placke, A. (1996) "Global distribution of species diversity in vascular plants: towards a world map of phytodiversity". Erdkunde 50, 1996, Vol.4, pp. 317-327. Available in: http://www.botanik.unibonn.de/phytodiv.htm.

Barthlott, W., Biedinger, N., Brau, G., Feig, F., Kier, G., Mutke, J. (1999) "Terminological and methodological aspects of the mapping and analysis of global biodiversity". Acta Botanica Fennica, 162 (1999), pp. 103-110.

Braschkat, J., Gärtner, S.O., Reinhardt, G.A. (2004) "Environmental impacts of bio-based products in comparison with conventional products". Agroindustria, Ed. Ranalli, 2004, Vol.2, pp. 53-59, Bologna.

Cowell, S.J. (1998) "Environmental Life Cycle Assessment of agricultural systems: integration into decision making". PhD dissertation, Centre of Environmental Strategy, University of Surrey, Guildford, UK.

Cowell, S.J., Clift, R. (2000) "A methodology for assessing soil quantity and quality in life cycle assessment". Journal of Cleaner Production, 8 (2000), pp. 321-331.

Gärtner, S.O., Reinhardt, G.A. (2004) "Biobased products and their environmental impacts with respect to conventional products". Proceedings of the 2nd World Conference on Biomass for Energy, Industry and Climate Protection, Rome. 
Goedkoop, M., Spriensma, R. (2001) "The Eco-indicator 99. A damage oriented method for Life Cycle Impact Assessment". Methodology report, Third Edition, PRé Consultants, Amersfoot, The Netherlands.

Känzig, J., Anex, R., Jolliet, O. (2003) "Conference report: International workshop on assessing the sustainability of bio-based products". International Journal of Life-Cycle Assessment, 2003, 8 (5), pp. 313-314.

Köllner, T. (2000) "Species-pool effect potentials (SPEP) as a yardstick to evaluate land-use impacts on biodiversity". Journal of Cleaner Production, 8 (2000), pp.293-311.

Köllner, T. (2001) "Land use in product life cycles and its consequences for ecosystem quality". Dissertation PhD 2519, Universität St. Gallen, Switzerland.

Lindeijer, E., van Kampen, M., Fraanje, P.J., van Gooben, H.F., Nabuurs, G.J., Schouwenberg, E.P.A.G., Prins, A.H., Dankers, N., Leopold, M.F. (1998) "Biodiversity and land use indicators for land use impacts in LCA". Ministerie VyW. Publicatiereeks Grondstoffen 1998/07, rapport n. W-DWW98-059, The Netherlands.

Lindeijer, E. (2000a) "Review of land use methodologies". Journal of Cleaner Production 8 (2000), pp.273-281.

Lindeijer, E. (2000b) "Biodiversity and life support impacts of land use in LCA". Journal of Cleaner Production, 8 (2000), pp. 313-319.

Lindeijer, E., Kok, I., Eggels, P., Alfers, A. (2002) "Improving and testing a land use methodology in LCA. Including casestudies on bricks, concrete and wood". Ed: Dutch Ministry of Transport, Public Works and Water Management (RWS DWW), The Netherlands.

Margalef, R. (1998) "Ecología”. ISBN 84-282-0405-5, Ed. Omega, Barcelona.

Mattsson, B., Cedeberg, C., Blix, L. (2000) "Agricultural land use in life cycle assessment (LCA): case studies of three vegetables oil crops". Journal of Cleaner Production, 8 (2000), pp. 283-292.

Milà i Canals, L. (2003) "Contributions to LCA methodology for agricultural systems. Site-dependency and soil degradation impact assessment". PhD dissertation, Universitat Autònoma de Barcelona, España.

Milà i Canals, L., Basson, L., Clift, R. Müller-Wenk, R., Bauer, C., Hansen, Y., Brandao, M. (2006) "Expert Workshop on Definition of Best Indicators for Biodiversity and Soil Quality for Life Cycle Assessment (LCA)". CES Working Paper 02/06, Centre for Environmental Strategy, ISSN: 1464-8083, University of Surrey, UK.
Müller-Sämann, K.M., Reinhardt, G.A., Vetter, R., Gärtner, S.O. (2003) "Nachwachsende Rohstoffe in BadenWürttemberg: Identifizierung Vorteilhafter Produktlinien zur stofflichen Nutzung unter Besonderer Berücksichtigung Unweltgerechter Anbauverfahren". Projektabschluss-bericht Forschungszentrum Karlsruhe IfUL Müllheim.

Müller-Wenk, R. (1998) "Land use - The main threat to species. How to include Land use in LCA". IWÖDiskussionbeitrag n.64, IWÖ, Universität St. Gallen, Switzerland.

Muys, B., García Quijano, J. (2002) "A new method for Land Use Impact Assessment in LCA based on the ecosystem exergy concept". Internal report, Laboratory for Forest, Nature and Landscape Research, KULeuven.

Patel, M., Bastioli, C., Marini, L., Würdinger, G.E. (2003) "Life-Cycle Assessment of Bio-Based Polymers and Natural Fibres". Encyclopedia "Biopolymers", 2003 Vol.10, pp. 409452.

Scout, G. (2000) "Green polymers". Polymer degradation and stability, 2000, 68, pp. 1-7.

van der Voet, E. (2001) "Land use in LCA". CML-SSP Working Paper, Centre of Environmental Science, Leiden University, The Netherlands.

Vitousek, P.M., Ehrlich, P.R., Ehrlich, A.H., Matson, P.A. (1986) "Human appropriation of the products of photosynthesis". Bioscience, Vol.36, n.6, 1986, pp. 368-373.

Vogtländer, J.G., Lindeijer, E., Witte, J.P.M., Hendriks, C. (2004) "Characterizing the change of land-use based on flora: application for EIA and LCA". Journal of Cleaner Production, 12 (2004), pp.47-57.

Wagendorp, T., Gulinck, H., Coppin, P., Muys, B. (2006) "Land use impact evaluation in life cycle assessment based on ecosystem thermodynamics". Energy, 31 (2006), pp. 112125.

Weidema, B., Lindeijer, E. (2001) "Physical impacts of land use in product life cycle assessment". Final report of the EURENVIRON-LCAGAPS sub-project on land use. Department of Manufacturing Engineering and Management, Technical University of Denmark. 